\title{
SIMILARITY JORDAN MULTIPLICATIVE MAPS
}

\author{
ZIJIE QIN AND FANGYAN LU
}

Abstract. We characterize bijections $\phi: B(X) \rightarrow B(X)$ satisfying that $\phi(A B+B A)$ and $\phi(A) \phi(B)+$ $\phi(B) \phi(A)$ are similar for all $A, B \in B(X)$.

Mathematics subject classification (2010): 47A30, 47B49.

Keywords and phrases: Jordan product, Jordan multiplicative map, similarity.

\section{REFERENCES}

[1] R. AN AND J. Hou, Additivity of Jordan multiplicative maps on Jordan operator algebras, Taiwanese J. Math. 10 (2006) 45-64.

[2] Z. BAI AND S. DU, Characterization of sum automorphisms and Jordan triple automorphisms of quantum probabilistic maps, J. Phys. A 43 (2010), 165210 (9 pp.).

[3] M. DoboviěK, B. KuzMa, G. LeŠnJak, C. K. Li And T. Petek, Mappings that preserve pairs of operators with zero triple Jordan product, Linear Algebra Appl. 426 (2007), 255-279.

[4] S. Du AND J. Hou, Nonlinear maps preserving similarity on B(X), Linear Algebra Appl. 420 (2007), $506-516$.

[5] A. E. Guterman And B. Kuzma, Preserving zeros of a polynomial, Communications in Algebra, 37 (2009), 4038-4064.

[6] F. HiAI, Similarity preserving linear maps on matrices, Linear Algebra Appl. 97 (1987), 127-139.

[7] J. Hou AND X. ZHANG, Additive maps preserving similarity of operator on Banach spaces, Act Math.Sin.(Engl.Ser.) 22 (2006), 179-186.

[8] G. Ji, Similarity-preserving linear maps on B(H), Linear Algebra Appl. 368 (2003), 249-257.

[9] SANG OG KIM AND C. PARK, Additivity of Jordan triple product homomorphisms on generalized matrix algebras, Bull. Korean Math. Soc. 50 (2013), 2027-2034.

[10] B. KUZMA, Jordan triple product homomorphisms, Monatsh. Math. 149 (2006), 119-128.

[11] G. LEŠNJAK AND N.-S. SZE, On injective Jordan semi-triple maps of $M_{n}$, Linear Algebra Appl. 414 (2006), 383-388.

[12] C. K. LI, P. ŠEMRL AND N.-S. SZE, Maps preserving the nilpotency of products of operators, Linear Algebra Appl. 424 (2007), 222-239.

[13] M. LiM, A note on similarity preserving linear maps on matrices, Linear Algebra Appl. 190 (1993), 229-233.

[14] F. LU, Additivity of Jordan maps on standard operator algebras, Linear Algebra Appl. 357 (2002), 123-131.

[15] F. Lu ANd C. Peng, Similarity-preserving linear maps on B(X), Studia Math. 209 (2012), 1-10.

[16] L. MOLNÁR, Non-linear Jordan triple automorphisms of sets of self-adjoint matrices and operators, Studia Math. 173 (2006), 39-48.

[17] T. PeteK, Linear mappings preserving similarity on $B(H)$, Studia Math. 161 (2004), 177-186.

[18] T. PETEK, A note on unitary similarity preserving linear mappings on $B(H)$, Linear Algebra Appl. 394 (2005), 217-224.

[19] T. PeteK, Unitary similarity preserving linear Maps on $B(H)$, Integral Equations Operator Theory 82 (2015), 51-60.

[20] Z. QIN AND F. LU, Involution similarity preserving linear maps, Studia Math. (to appear)

[21] P. ŠEMRL, Maps on idempotent matrices over division rings, J. Algebra 298 (2006), 142-187.

[22] P. ŠEmRL, Maps on idempotents, Studia Math. 169 (2005), 21-44. 
[23] P. ŠEMRL, Similarity preserving linear maps, J. Operator Theory 60 (2008), 71-83.

[24] Z. Yang AND F. Lu, Surjective Jordan maps and Jordan triple maps, Linear Algebra Appl. 535 (2017), 1-11.

[25] L. ZHAO AND J. Hou, Jordan zero-product preserving additive maps on operator algebras, J. Math. Anal. Appl. 314 (2006), 689-700. 